\title{
Achieving Directionality and Transmit Diversity via Integrating Beam Pattern Scanning (BPS) Antenna Arrays and OFDM
}

\author{
Peh Keong Teh, Seyed Alireza Zekavat \\ Department of Electrical and Computer Engineering, Michigan Technological University, Houghton, USA \\ E-mail: \{pteh,rezaz\}@mtu.edu \\ Received July 28, 2009; revised October 25, 2009; accepted November 19, 2009
}

\begin{abstract}
In this paper, we introduce a novel merger of antenna arrays with scanning beam patterns, and Orthogonal Frequency Division Multiplexing (OFDM) systems. Controlled time varying phase shifts are applied to the antenna array elements mounted at the base station with beam patterns directed toward the desired user. This creates a small beam pattern movement called Beam Pattern Scanning (BPS). In rich scattering environments BPS creates a time varying environment leading to time diversity exploitable at the receiver enhances its probability-of-error performance. Here, we apply OFDM signals to BPS antenna arrays, and we achieve: (1) directionality, which supports Space Division Multiple Access (SDMA); and (2) a time diversity gain, which leads to high performance. We discuss the structure of the base station antenna array and the OFDM receiver that exploits time diversity. We also introduce the merger of BPS and multi-carrier OFDM (MC-OFDM) systems. In MC-OFDM each bit is transmitted over all sub-carriers after serial to parallel conversion. BPS/ MC-OFDM receiver exploits both time diversity inherent in BPS, and frequency diversity inherent in MC-OFDM transmission technique. Simulation results show high Probability-of-error performance is achievable via BPS/OFDM and BPS/MC-OFDM schemes comparing to the traditional OFDM and MC-OFDM, respectively. Simulations also reveal that MC-OFDM system as well as its merger with BPS is capable of mitigating large Peak-to-Average Ratio (PAPR) problem in traditional OFDM system. In addition, performance simulations with coded OFDM (COFDM) and coded MC-OFDM (MC-COFDM) and their merger with BPS are studied.
\end{abstract}

Keywords: OFDM, MC-OFDM, Antenna Array, Beam Pattern Scanning, Transmit Diversity.

\section{Introduction}

OFDM (Orthogonal Frequency Division Multiplexing) is an emerging technique capable of high data rate transmission over frequency selective channels without implementation of complex equalizers [1-5]. Due to its inherent benefits, OFDM has been proposed as the basic modulation technique for the 4th generation wireless systems. In an $N$ sub-carriers OFDM system, a block of $N$ information bits are serial to parallel converted and modulated via $N$ orthogonal sub-carriers (each bit over one sub-carrier). They are then summed and transmitted simultaneously [1-5]. This process extends the symbol duration from $T_{s}$ to $N T_{s}$.

MC-OFDM (Multi-Carrier OFDM) is an innovative OFDM transmission technique where each MC-OFDM user's bit is transmitted over all available sub-carriers simultaneously [6-8]. To ensure separability of the bits at the receiver and reduce inter-bit-interference (IBI), orthogonal codes, e.g., Hadamard Walsh codes, are applied to the sub-carriers of each bit. Through MC-OFDM, frequency diversity can be exploited to improve the performance of the system with minimal complexity in the transmitter and receiver $[7,8]$.

Properly designed OFDM and MC-OFDM systems convert the channel to a flat fading channel and eliminate the need for complex equalizers. This is achieved via selecting $N$ such that $N T_{s}$ becomes much larger than channel delay spread. However, there is a drawback associated with flat fading: If sub-carriers experience deep fade, the bit will be rendered unrecoverable and thereby degrades the system probability-of-error performance. This issue can be resolved via diversity techniques. In MCOFDM signals are transmitted over all sub-carriers, 
which allow frequency diversity to be exploited at the receiver [6-8]. Besides frequency diversity, various methods such as: 1) Forward Error Correction Coding at the cost of the overall system throughput [1,2], and 2) transmit diversity has been used to improve the probabilityof-error performance of OFDM and MC-OFDM systems $[9,10]$. In this work, we merge OFDM systems with a transmit diversity scheme created via beam pattern movement (scanning).

The concept of beam pattern movement has been referenced in the literature, some with a different approach, and some with no further exploration. For example, in [11], researchers introduce "jitter diversity". In that work, with a high angular spread at the mobile receiver, the beam pattern is jittered around its usual position to create angle diversity and enhance the receiver performance; and it does not lead to time diversity. In [12], there is a very short (just one line) reference to the idea of antenna pattern movement to create time diversity. The authors state that one can force the antenna to oscillate when the vehicle is traveling at low speeds, channel's fade is slow, and time diversity cannot be exploited.

Recently, a powerful transmit diversity technique has been introduced called beam pattern scanning (BPS) (also known as beam pattern oscillation). In this scheme, antenna arrays are installed at the base station (BS) with their scanning (oscillating) antenna patterns directed toward the desired users [13,15]. A time varying phase shift is applied to each antenna element in order to steer and move the antenna pattern within one symbol duration $T_{s}$ (or within $N T_{s}$ in OFDM systems). The beam pattern starts sweeping an area of space at time zero, it returns to its initial position after time $T_{s}\left(N T_{s}\right.$ in OFDM) and repeats its sweeping. The movement of the beam pattern is small, e.g., in the order of $5 \%$ of half power beam width (HPBW). Hence, the desired user stays in the antenna array HPBW at all times.

In rich scattering environments, as a result of the departure and arrival of scatterers within the window of antenna beam pattern, BPS creates a time varying channel with a small controlled coherence time $T_{c}$ with respect to $T_{s}\left(N T_{s}\right)[8,13,14]$. This leads to a fast fading channel via which time diversity benefits can be exploited at the receiver [16]. Therefore, BPS is introduced as a transmit diversity scheme that enhances: a) receiver probability-of-error performance via time diversity, and b) wireless network capacity via Spatial Division Multiple Access (SDMA) [17] or spatial filtering interference reduction (SFIR) [18].

In this paper, we merge BPS transmit diversity with OFDM and MC-OFDM systems. This merger achieves: 1) high probability-of-error performance by time diversity induced at the receiver via BPS $[8,15,19]$, and 2) high capacity via directionality created via antenna arrays mounted at the transmitter [17-20], with a 3) low complexity due to the structure of OFDM and BPS. The structure of BPS is simple because the complexity is mainly focused at the base station antenna array.

In this work, we present and discuss: a) the antenna array structure that makes BPS possible for OFDM systems incorporating a number of sub-carriers, b) the OFDM receiver structure capable of exploiting the time diversity induced by BPS, and c) the MC-OFDM receiver capable of exploiting time diversity via BPS and frequency diversity through Multi-carrier scheme. We simulate the probability-of-error performance and the peak-to-average ratio (PAPR) curves for both BPS/OFDM and BPS/MCOFDM.

Traditional OFDM and MC-OFDM utilizing antenna arrays without BPS scheme are used as benchmark against BPS/OFDM and BPS/MC-OFDM schemes. Adaptive antenna arrays with beam patterns directed towards intended users leads to capacity enhancement via SDMA [20-22] without enhancing the performance. This paper highlights performance benefits achieved through BPS and OFDM systems (OFDM and MC-OFDM) merger. In addition, coded version of OFDM systems and BPS merged systems (BPS/COFDM and BPS/MC-COFDM) are simulated and compared to further underline the performance improvement through BPS merger.

Section 2 introduces OFDM and MC-OFDM systems, BPS technique and the antenna array structure. In Section 3, we present BPS/OFDM and BPS/MC-OFDM received signal and their receiver structure. In Section 4, we present the probability-of-error performance and peak-to-average ratio simulations. Section 5 concludes the paper.

\section{OFDM, MC-OFDM, Antenna Array Structure and BPS Techniques}

1) OFDM system: In OFDM, a block of $N$ bits are transmitted simultaneously over $N$ sub-carriers (each bit over one sub-carrier) after serial to parallel conversion, which converts the duration of transmitted symbols from $T_{s}$ to $N T_{s}$ (see Figure 1). The number of sub-carriers $N$ is chosen to ensure flat fading channel at all times (i.e., channel delay spread $T_{m}<<N T_{s}$ ), and the sub-carriers are separated by

$$
\Delta f=1 / N T_{s}
$$

to ensure the orthogonality of sub-carriers and avoid inter-carrier interference at the receiver. The OFDM transmitted signal corresponds to:

$$
s_{i}(t)=\operatorname{Re}\left\{\sum_{n=0}^{N-1} b[n+i N] e^{j 2 \pi\left(f_{o}+n \Delta f\right) t}\right\} \cdot g_{N T_{s}}\left(t-i N T_{s}\right)
$$

where $b[\cdot] \in\{+1,-1\}$ is the transmitted bit, $i \in\{0,1,2, \ldots\}$ is the $i^{\text {th }}$ group of $N$ bits simultaneously converted to par- 


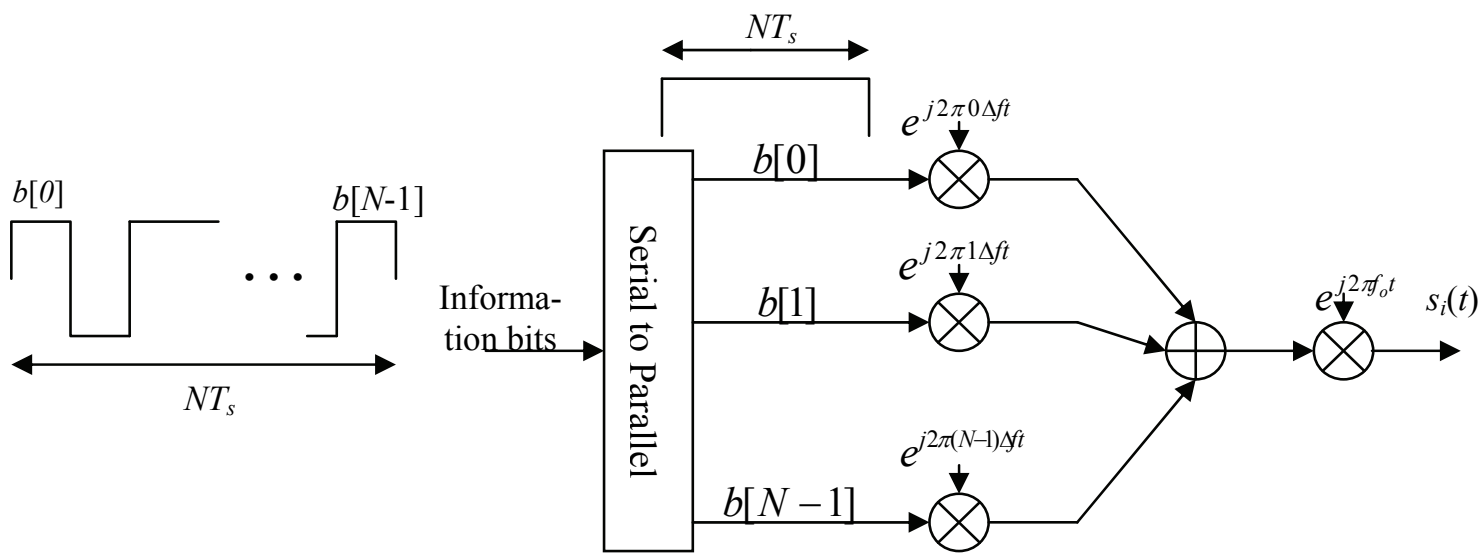

Figure 1. OFDM transmitter structure.

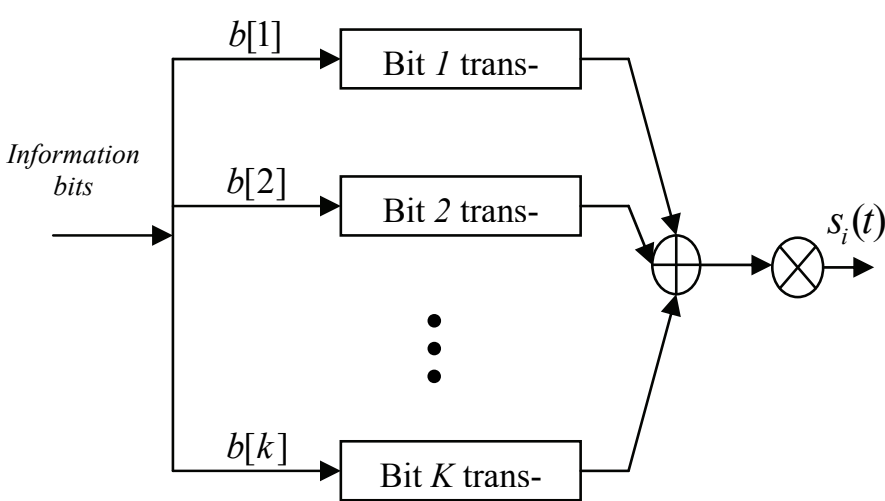

(a)

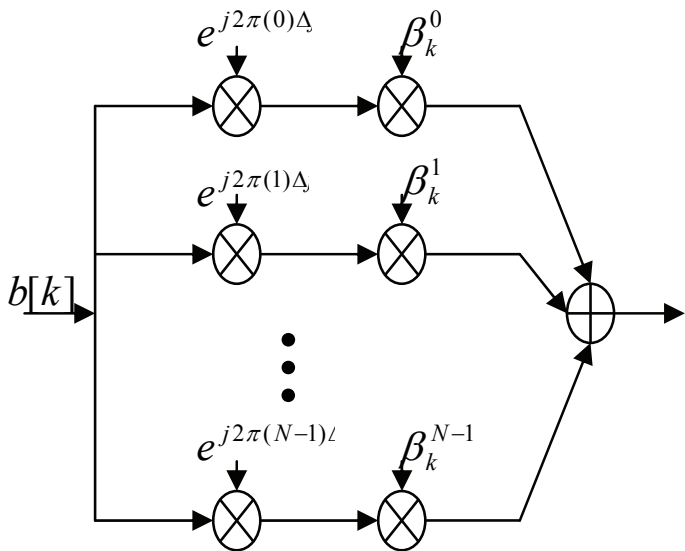

(b)

Figure 2. (a) MC-OFDM transmitter structure; (b) $k^{\text {th }}$ bit transmitter design.

allel ( $n$ represents the bit number), $f_{o}$ is the carrier frequency and $g_{N T_{s}}(t)$ is a rectangular waveform of unity height over zero to $N T_{s}$ ( $T_{s}$ refers to symbol duration).

2) The MC-OFDM system: In MC-OFDM system, all $N$ bits are transmitted simultaneously over all $N$ subcarriers. To maintain orthogonality across bits, an orthogonal code, e.g., Hadamard Walsh code, is assignedto the sub-carrier of each bit (see Figure 2). By transmitting all $N$ bits simultaneously, the symbol duration is converted from $T_{s}$ to $N T_{s}$. Hence, the MC-OFDM transmitted signal corresponds to:

$s_{i}(t)=\operatorname{Re}\left\{\sum_{k=0}^{N-1} \sum_{n=0}^{N-1} b[k+i N] \cdot \beta_{k}^{n} \cdot e^{j 2 \pi\left(f_{o}+n \Delta f\right) t}\right\} \cdot g_{N T_{s}}\left(t-i N T_{s}\right)$

where $\beta_{k}^{n}$ is the $k^{\text {th }}$ bit and $n^{\text {th }}$ sub-carrier orthogonal codes' element. Other parameters have been defined in (2).
3) Proposed Antenna Array Structure: We assume an $M$-element linear antenna array mounted at the Base Station (B.S.) (Figure 3). In order to move the antenna pattern, a time varying phase, $m \theta(t)$, is applied to the $m^{\text {th }}$ antenna array element (Figure 3). Moreover, in Figure 3 the angle $\phi_{o}$ represents the direction of the antenna beam pattern (angle-of-arrival of the signal) with respect to the antenna array main axis. Here, for simplicity in presentation, it is assumed that the desired mobile is located at angle $\phi_{o}=0$ (i.e., antenna array main axis and beam pattern main axis are overlapped). In this case, the normalized array factor characterizing the resulting antenna pattern corresponds to:

$$
A F(t, \phi)=\frac{1}{M}\left[\frac{\sin \left(\frac{M}{2} \gamma(t, \phi)\right)}{\sin \left(\frac{1}{2} \gamma(t, \phi)\right)}\right],
$$

where

$$
\gamma(t, \phi)=\frac{2 \pi}{\lambda_{o}} d \cdot \sin \phi-\theta(t)
$$


Here, $\lambda_{o}$ is the wavelength $\left(c / f_{o}\right), d$ is the distance between antenna elements, and $\left(2 \pi d / \lambda_{0}\right) \cdot \sin \phi$ represents the phase offset due to the difference in distance between antenna array elements and the mobile.

In general, antenna array half power beam width (HPBW) changes with frequency (sub-carrier). For narrowband OFDM systems, the variation in HPBW is negligible (see Figure 4); however, for wideband and high data rate OFDM systems, e.g., $60 \mathrm{Mb} / \mathrm{s}$ data rate, the variation of beam pattern with sub-carriers is considerable (see Figure 4). In order to achieve directionality via sectoring strategy (e.g., in switched beam smart antenna systems), identical beam patterns are required for all $N$ sub-carriers. Since different sub-carriers create different HPBWs, users located near the border of two sectors may experience either: 1) large interference from the beam pattern of unintended sector, or 2) a reduction in power in the desired signal in the beam pattern from the desired sector [15].

We must then ensure that the size of sub-carriers applied to antenna array is small enough that all the sub-carriers placed in the antenna array generate identi- cal patterns. Hence, we introduce the following definition:

Definition 1: Beam Patterns are considered identical if the variation in null-to-null beam width $(\Delta \mathrm{B}$.W. in Figure 4) of the beam patterns created via sub-carriers are within $10 \%$ of the average null-to-null beam width (B.W.) $)_{\text {ave }}$. Based on this definition, it can be shown via simulations that whenever the minimum frequency, $f_{\min }$, and the maximum frequency, $f_{\max }$, applied to an antenna array satisfy

$$
\frac{f_{\text {max. }}-f_{\text {min. }}}{f_{o}}<0.01,
$$

"identical" beam patterns are observed (see [15]). Considering a contiguous bandwidth for OFDM systems, this criterion corresponds to

$$
N \cdot \Delta f / f_{o}<0.01
$$

If (6) is not satisfied, the $N$ sub-carriers of the OFDM system should be divided into $P$ groups of $Q \leq N(N=P Q)$ neighboring sub-carriers such that $Q \Delta f_{d} f \leq 0.01$. Then, either each set of $Q$ sub-carriers should be applied into a

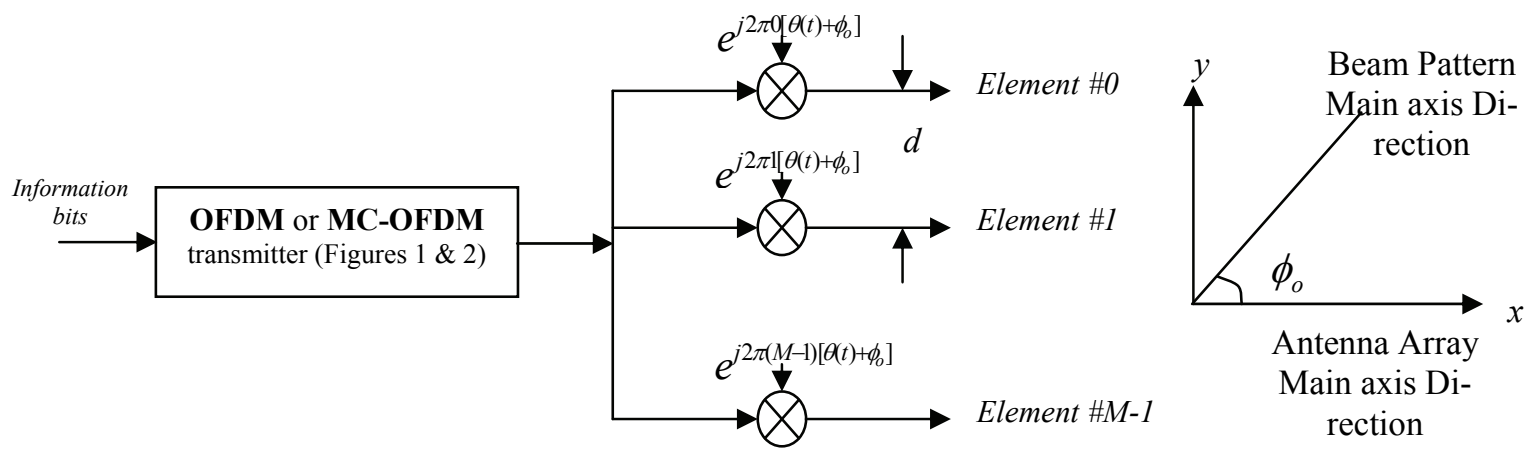

Figure 3. Antenna structure.

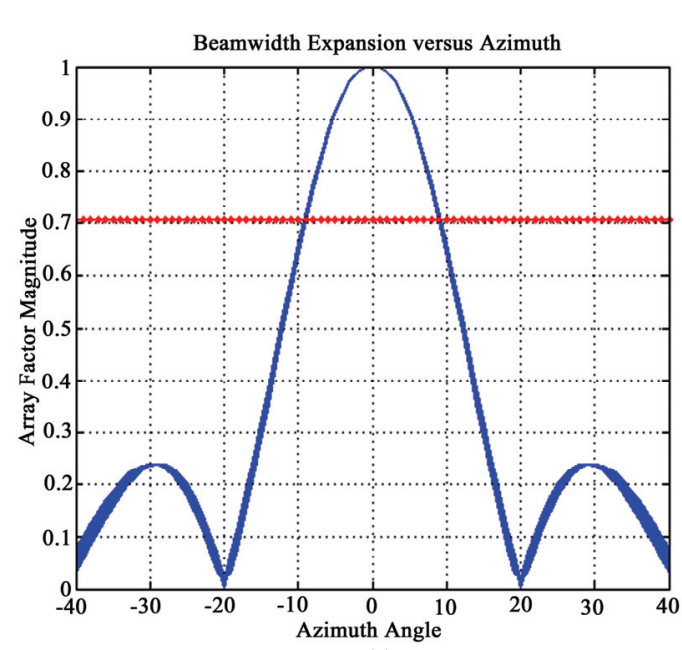

(a)

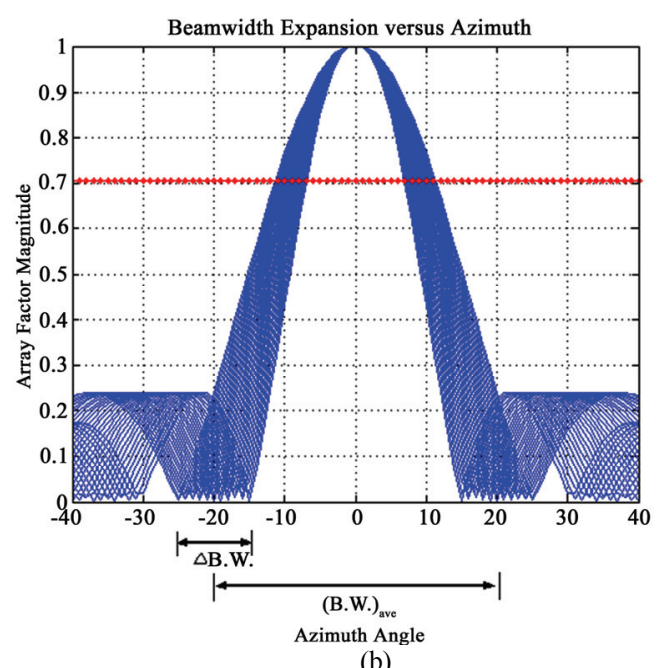

(b)

Figure 4. 32 sub-carriers OFDM system, center frequency 4.0GHz. (a) Narrow band system with bandwidth of $200 \mathrm{MHz}$; (b) Wide band system with bandwidth of $2 \mathrm{GHz}$. 
unique $M$-element antenna array or unique complex weights should be applied to antenna elements for each set of sub-carriers $[15,17,19]$. In this work, we assume a narrow band system with $Q=N, P=1$, i.e., all of sub-carriers, $f_{n}=f_{o}+n \Delta f, n \in\{0,1, \ldots, N-1\}$ are applied to one antenna array (see Figure 3).

4) Beam Pattern Scanning Technique: The scanning of beam pattern is created at the B.S. by applying time varying phase offsets $m \theta(t)$ to the antenna array element $m \in\{1,2, \ldots, M\}$ (Figure 3). The antenna array's beam pattern movement should ensure: 1) constant large scale fading, and 2) $L$ independent fades to be generated within each $N T_{s}$. This creates an $L$-fold diversity gain at the mobile receiver. After each $N T_{s}, \theta(t)$ returns the beam pattern to its $t=0$ position and repeats an identical spatial sweeping movement over the next $N T_{s}$ period (Figure 5(a)).

In order to ensure constant large-scale fading, the mobile must remain within the antenna array's HPBW over each symbol duration $N T_{s}$. This condition corresponds to [22].

$$
\left|N T_{s} \frac{d \phi}{d t}\right|=\kappa \cdot B, \quad 0<\kappa<1
$$

where $B$ is the HPBW, $\phi$ is the azimuth angle (direction of arrival), $d \phi / d t$ is the rate of antenna pattern movement, and $N T_{s} \cdot(d \phi / d t)$ is the amount of antenna pattern movement within $N T_{s}$. The parameter $\kappa, 0<\kappa<1$, is the control parameter ensures the received antenna pattern amplitude is within the HPBW for the entire symbol duration. The phase offset applied to the antenna array is calculated using (8) leads to [9] (see Figure 5(b)):

$$
\theta(t)=\kappa \cdot \frac{2 \pi d \cdot|\cos \phi| \cdot B}{\lambda_{o} N T_{s}} \cdot\left(t-\frac{N T_{s}}{2}\right)
$$

Specifically, to solve for (9) from (8) (detailed in [20]), we proceed as follows: 1) Using (5), we solve for $\phi$ at a fixed value of $\left.\chi(t, \phi)=\gamma_{o} ; 2\right)$ substituting this $\phi$ value into (8) and differentiating, we create a differential equation for $\theta(t)$; and (3) finally, we solve the differential equation which leads directly to (9).

Sweeping of the antenna pattern creates a time-varying channel with a coherence time that is a function of scattering environment and may lead to $L$ independent fades over $N T_{s}$. Geometric-based stochastic channel modeling scheme is used to evaluate the channel diversity gain [11]. Channel coherence time calculated in [15] assuming a medium size city center (e.g., 3 scatterers per $1000 \mathrm{~m}^{2}$ ), with $0.0005<\kappa<0.05$ shows that diversity gain as high as $L \approx 7$ is achievable via BPS scheme. The main assumption in this modeling scheme is:

1) A semi-elliptical coverage with the mobile at its center (suitable when we assume the height of the BS antenna array is close to the height of surrounding buildings);

2) The movement generated by antenna array oscillation is dominant, and hence the movement of mobile and other relative speed object in the environment is ignored;

3) Scatters are assumed to have dimensions in accordance with a known PDF [13];

4) Scatters in the surrounding BS are uniformly distributed;

5) Scatters are consider diffused reflectors which reflect the incident radiation in all direction; and,

6) The signal received at the mobile is sum of horizontally propagated plane waves interacting with just one scatterer.

\section{Receiver Design}

1) BPS/OFDM: For simplicity in presentation, we consider $i=0$ in (2). We represent the OFDM transmitted signal as:

$s_{0}(t)=\sum_{n=0}^{N-1} b[n] \cos \left(2 \pi\left(f_{o}+n \Delta f\right) t\right), \quad t \in\left[0, N T_{s}\right]$

Applying this signal to the antenna array in Figure 3 the output of the $m^{\text {th }}$ element of the antenna array is

$$
s_{m}(t)=\sum_{n=0}^{N-1}\left[b[n] \cos \left(2 \pi\left(f_{o}+n \Delta f\right) t+m \theta(t)\right)\right], \quad t \in\left[0, N T_{s}\right]
$$

The total normalized downlink transmitted signal, considering all antenna elements (all $\mathrm{m}$ ) is:

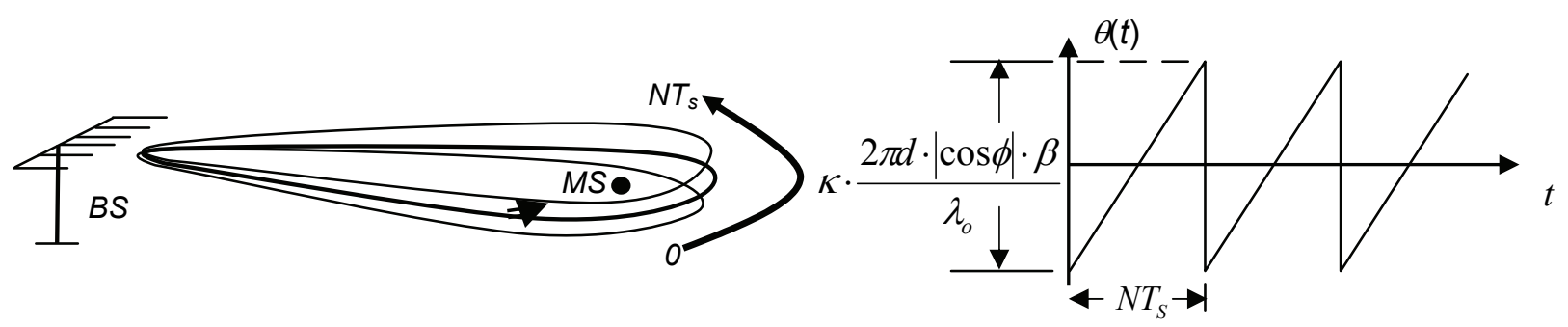

(a)

(b)

Figure 5. (a) Beam pattern scanning; (b) Antenna element delay line function. 


$$
s(t)=\sum_{n=0}^{N-1} b[n] \frac{1}{M} \sum_{m=0}^{M-1} \cos \left(2 \pi\left(f_{o}+n \Delta f\right) t+m \theta(t)\right) t \in\left[0, N T_{s}\right]
$$

Since the range of the control parameter is taken $0<\kappa$ $<0.05$ in (8), the frequency offset induced by $\theta(t)$ in the transmitted signal is less than $5 \%$ and can be ignored. Typically, as it is seen in (9), assuming half wavelength spacing across antenna elements, and $N=128$, and $B \cong \pi$ / 6 (for 4 element antenna), and $T_{s}=10^{-6}$, the maximum frequency drift would be in the order of $100 \mathrm{~Hz}$, which is minimal compared to the typical carrier frequency of 2.4 $\mathrm{GHz}$. At the receiver side, considering the transmit diversity leads to $L$-fold time diversity, the received signal $\left[0, N T_{s}\right]$ can be divided into time slots $\left[l N T_{s} / L,(l+1) N T_{s} / L\right]$, $l \in\{0,1, \ldots, L-1\}$, and these time slots demonstrate independent fades. The received signal can then be represented as

$$
\begin{aligned}
& r_{l}(t)=\frac{1}{L} \sum_{n=0}^{N-1} \alpha_{l}^{n} b[n] \cdot \frac{1}{M} \cdot \sum_{m=0}^{M-1} \cos \left(2 \pi\left(f_{o}+n \Delta f\right) t\right. \\
& \left.+m \gamma(t, \phi)+\xi_{l}^{n}\right)+n_{l}(t) \quad t \in\left[l N T_{s} / L,(l+1) N T_{s} / L\right]
\end{aligned}
$$

where, $n_{l}(t)$ is an additive white Gaussian noise (AWGN), which is independent for different time slots $(l), \alpha_{l}^{n}$ is the Rayleigh fade amplitude on $n^{\text {th }}$ sub-carrier in the $l^{\text {th }}$ time slots, and $\xi_{l}^{n}$ is the fading phase offset in the $n^{\text {th }}$ sub-carrier and $l^{\text {th }}$ time slot (hereafter, this phase offset is assumed to be tracked and removed). The Rayleigh fade amplitudes are considered independent over time and correlated over sub-carriers. The correlation coefficient between carrier $n^{\prime}$ and $n^{\prime \prime}$ is characterized by [23]

$$
p_{n^{\prime}, n^{\prime \prime}}=\frac{1}{1+\left(\left(n^{\prime \prime}-n^{\prime}\right) \cdot\left(\Delta f /(\Delta f)_{c}\right)\right)^{2}}
$$

where $(\Delta f)_{c}$ is the coherence bandwidth of the channel. Moreover, $\gamma(t, \phi)$ in (13) is introduced in (5). Applying the summation over $m$, (13) corresponds to

$$
\begin{aligned}
& r_{l}(t)=\sum_{n=0}^{N-1} \alpha_{l}^{n} \cdot b[n] \cdot A F(t, \phi) \cdot \cos \\
& \left(2 \pi\left(f_{o}+n \Delta f\right) \cdot t+\frac{M-1}{2} \gamma(t, \phi)\right)+n_{l}^{n}(t)
\end{aligned}
$$

Here, $A F(t, \phi)$ introduced in (4), is the normalized antenna array factor. Assuming a narrow-beam width antenna array and the mobile is located at $\phi_{o}=0,(5)$ can be approximated by $\chi(t, \phi)=\gamma(t)=\theta(t)$. Assuming that antenna array peak directed towards the intended mobile at time 0 , and with small movements of antenna array pattern over $N T_{s}$, the array factor experience over $\left[0, N T_{s}\right]$ is well approximated by $A F(t, \phi) \cong 1$.

The BPS/OFDM receiver is shown in Figure 6. In this figure, "Re" refers to Real Part, and "Im" refers to Imaginary Part. In addition, after the application of

$$
v=\sqrt{\frac{1}{2 N T_{s}}} e^{-j\left(2 \pi f_{o} \cdot t+\frac{M-1}{2} \gamma(t)\right)}
$$

and returning the OFDM to baseband, a bank of bandpass filters are used to separate the OFDM signal into its $N$ multiple sub-carriers: The baseband signal is integrated over each interval of $t \in\left[l N T_{s} / L,(l+1) N T_{s} / L\right], l \in$ $\{0,1, \ldots, L-1\}$ in order to exploit time diversity components created by beam pattern scanning $[14,15]$. The received signal for each subcarrier, $n$ and $l$ time slot corresponds to

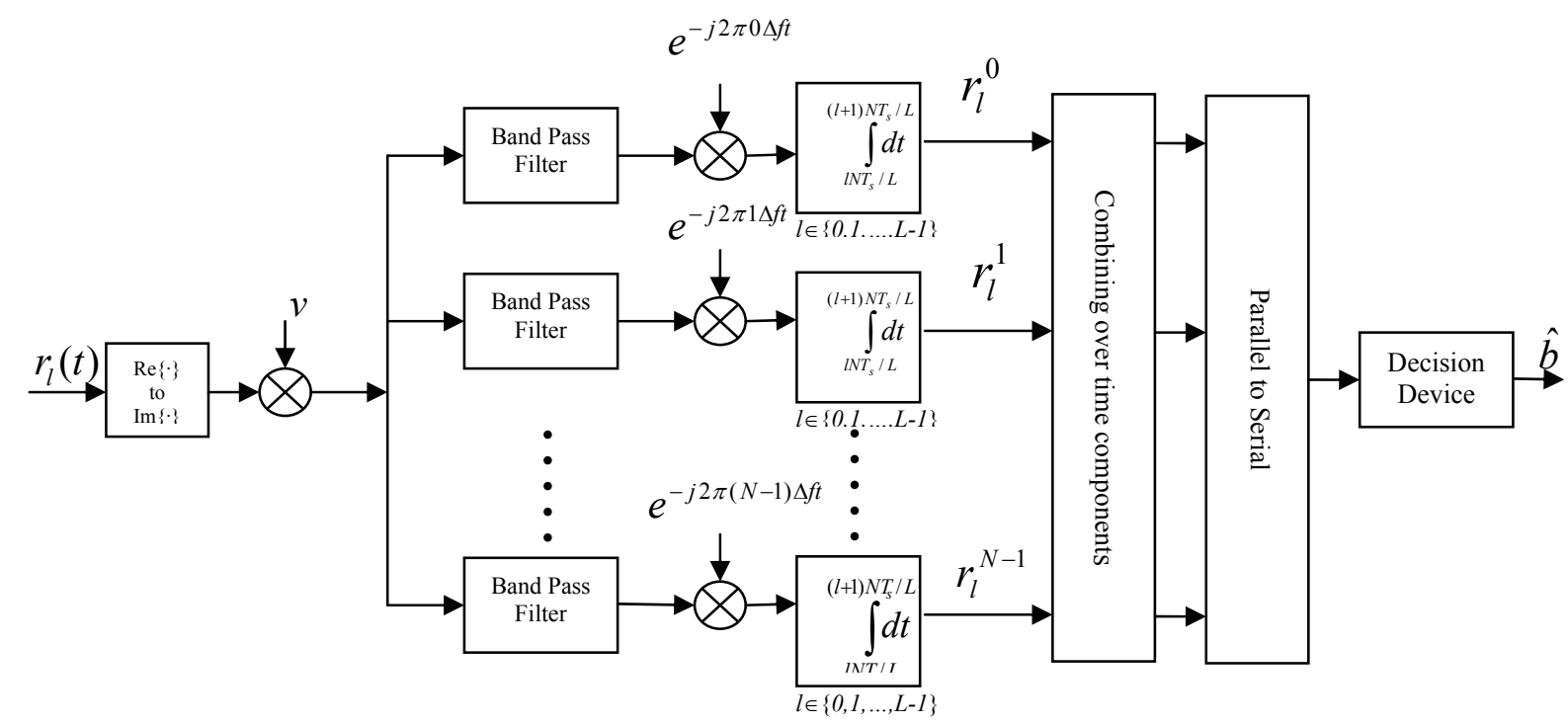

Figure 6. BPS-OFDM receiver. 


$$
\begin{gathered}
r_{l}^{n}=\frac{1}{L} \sqrt{\frac{N T_{s}}{2}} \alpha_{l}^{n} \cdot b[n]+n_{l}^{n}, n \in \\
\{0,1, \ldots, N-1\}, l \in\{0,1, \ldots, L-1\}
\end{gathered}
$$

where $n_{l}^{n}$ is a zero-mean Gaussian random variables with variance $N_{o} / 2$. The first term in (17) represents $l^{\text {th }} \times$ $n^{\text {th }}$ component of the desired signal and the second term is noise. With $N \times L$ diversity components, $N$ over frequency and $L$ over time, the combiner can be design to utilize different combining techniques such as Equal Gain Combining (EGC) or Maximum Ratio Combining (MRC) (in single user environment, Minimum Mean Square Error Combining (MMSEC) leads to a similar result as MRC).

2) $B P S / M C-O F D M$ : Here, we consider $i=0$ in (3) and we ignore pulse shaping function $g_{N T_{s}}(t)$. The MC-OFDM transmitted signal corresponds to

$$
s_{0}(t)=\sum_{k=0}^{N-1} \sum_{n=0}^{N-1} b[k] \cdot \beta_{k}^{n} \cdot \cos \left(2 \pi\left(f_{o}+n \Delta f\right) t\right), t \in\left[0, N T_{s}\right]
$$

This signal is applied to the antenna array in Figure 3 and the output of the $m^{\text {th }}$ element of the antenna array is given by,

$$
\begin{aligned}
& s_{m}(t)=\sum_{k=0}^{N-1} \sum_{n=0}^{N-1}\left[b[k] \cdot \beta_{k}^{n} \cdot \cos \left(2 \pi\left(f_{o}+n \Delta f\right) t+m \theta(t)\right)\right] \\
& t \in\left[0, N T_{s}\right], m \in\{0,1, \ldots, M-1\}
\end{aligned}
$$

Considering all antenna elements (all $\mathrm{m}$ ), the total normalized downlink transmitted signal is

$$
\begin{aligned}
& s(t)=\sum_{k=0}^{N-1} \sum_{n=0}^{N-1} b[n] \cdot \beta_{k}^{n} \cdot \frac{1}{M} \sum_{m=0}^{M-1} \cos \left(2 \pi\left(f_{o}+n \Delta f\right) t+m \theta(t)\right) \\
& t \in\left[0, N T_{s}\right]
\end{aligned}
$$

Again, in (20) the frequency offset induced by $\theta(t)$ in the transmitted signal is minimal and it is ignored, since the range of the control parameter $\kappa<0.05$ in (6) creates less than $5 \%$ bandwidth expansion. At the receiver, since the transmit diversity leads to an $L$-fold time diversity, the received signal can be divided into time slots $\left[l N T_{s} / L\right.$, $\left.(l+1) N T_{s} / L\right], l \in\{0,1, \ldots, L-1\}$ and each individual slot demonstrates independent fades. The received signal can be represented as

$$
\begin{aligned}
& r_{l}(t)=\sum_{k=0}^{N-1} \sum_{n=0}^{N-1} \alpha_{l}^{n} b[n] \cdot \beta_{k}^{n} \cdot \frac{1}{M} \cdot \sum_{m=0}^{M-1} \cos \left(2 \pi\left(f_{o}+n \Delta f\right) t+\right. \\
& \left.m \gamma(t, \phi)+\xi_{l}^{n}\right)+n_{l}(t) \quad t \in\left[l N T_{s} / L,(l+1) N T_{s} / L\right]
\end{aligned}
$$

where, $n_{l}(t)$ is an additive white Gaussian noise (AWGN), which is considered independent for different time slots (l), $\alpha_{l}^{n}$ is the Rayleigh fade amplitude on the $n^{\text {th }}$ sub-carrier in the $l^{\text {th }}$ time slots, and $\xi_{l}^{n}$ is the fading phase offset in the $n^{\text {th }}$ sub-carrier in the $l^{\text {th }}$ time slot (hereafter, this phase offset is assumed to be tracked and removed). The Rayleigh fade amplitudes, $\alpha_{l}^{n}$, are independent over time $(l)$ and correlated over sub-carriers $(n)$ with the correlation coefficient between sub-carriers $n^{\prime}$ and $n^{\prime \prime}$ characterized by (14). Applying the summation over $m$, (21) corresponds to

$$
\begin{aligned}
r_{l}(t) & =\sum_{k=0}^{N-1} \sum_{n=0}^{N-1} \alpha_{l}^{n} \cdot b[n] \cdot \beta_{k}^{n} \cdot A F(t, \phi) \cdot \cos \left(2 \pi\left(f_{o}+n \Delta f\right) \cdot t\right. \\
& \left.+\frac{M-1}{2} \gamma(t, \phi)\right)+n_{l}(t)
\end{aligned}
$$

The structure of BPS/MC-OFDM receiver is shown in Figure 7 (Figure 7(b) represents jth bit receiver). The received signal for each sub-carrier, $n$ and time slot $l$ can be represented by

$$
\begin{aligned}
& r_{l}^{n}[j]=\frac{1}{L} \sqrt{\frac{N T_{s}}{2}} \alpha_{l}^{n} \cdot b[j]+\frac{1}{L} \sum_{\substack{k=0 \\
k \neq j}}^{N-1}\left[\sqrt{\frac{N T_{s}}{2}} \alpha_{l}^{n} \cdot b[k] \cdot \beta_{k}^{n} \cdot \beta_{j}^{n}\right] \\
& +n_{l}^{n}, n \in\{0,1, \ldots, N-1\}, l \in\{0,1, \ldots, L-1\}
\end{aligned}
$$

where $n_{l}^{n}$ is a zero-mean Gaussian random variables with variance $N_{o} / 2$. In (23), the first term represent the desired signal, the second term represents the inter-bit-interference and the third term represents the noise. The factor $1 / L$ is the direct consequence of dividing the received signal into $L$ partitions creating $L$-fold time diversity. The combiner can be designed to utilize different combining techniques in frequency and time domain. Different combining methods in frequency domain are:

1) Equal Gain Combining (EGC)

$$
R_{l, E G C}=\sum_{n=0}^{N-1} r_{l}^{n}
$$

2) Maximum Ratio Combining (MRC)

$$
R_{l, M R C}=\sum_{n=0}^{N-1} \alpha_{l}^{n} \cdot r_{l}^{n}
$$

3) Minimum Mean Square Error Combining (MMSEC)

$$
R_{l, M M S E C}=\sum_{n=0}^{N-1} r_{l}^{n} \cdot\left[\frac{\alpha_{l}^{n}}{N\left(\alpha_{l}^{n}\right)^{2}+N_{0} / 2}\right]
$$

Here, we present only Equal Gain Combining (EGC) in the time domain to exploit the diversity induced by BPS.

\section{Simulated Performance}

Simulations are provided for MC-OFDM systems with antenna arrays. The assumptions for these simulations are as follow: 1) $N=32$ sub-carriers in the MC-OFDM system; 2) $L=7$ independent fades are achievable as a result of the beam-pattern movement in the duration $N T_{s}$ 


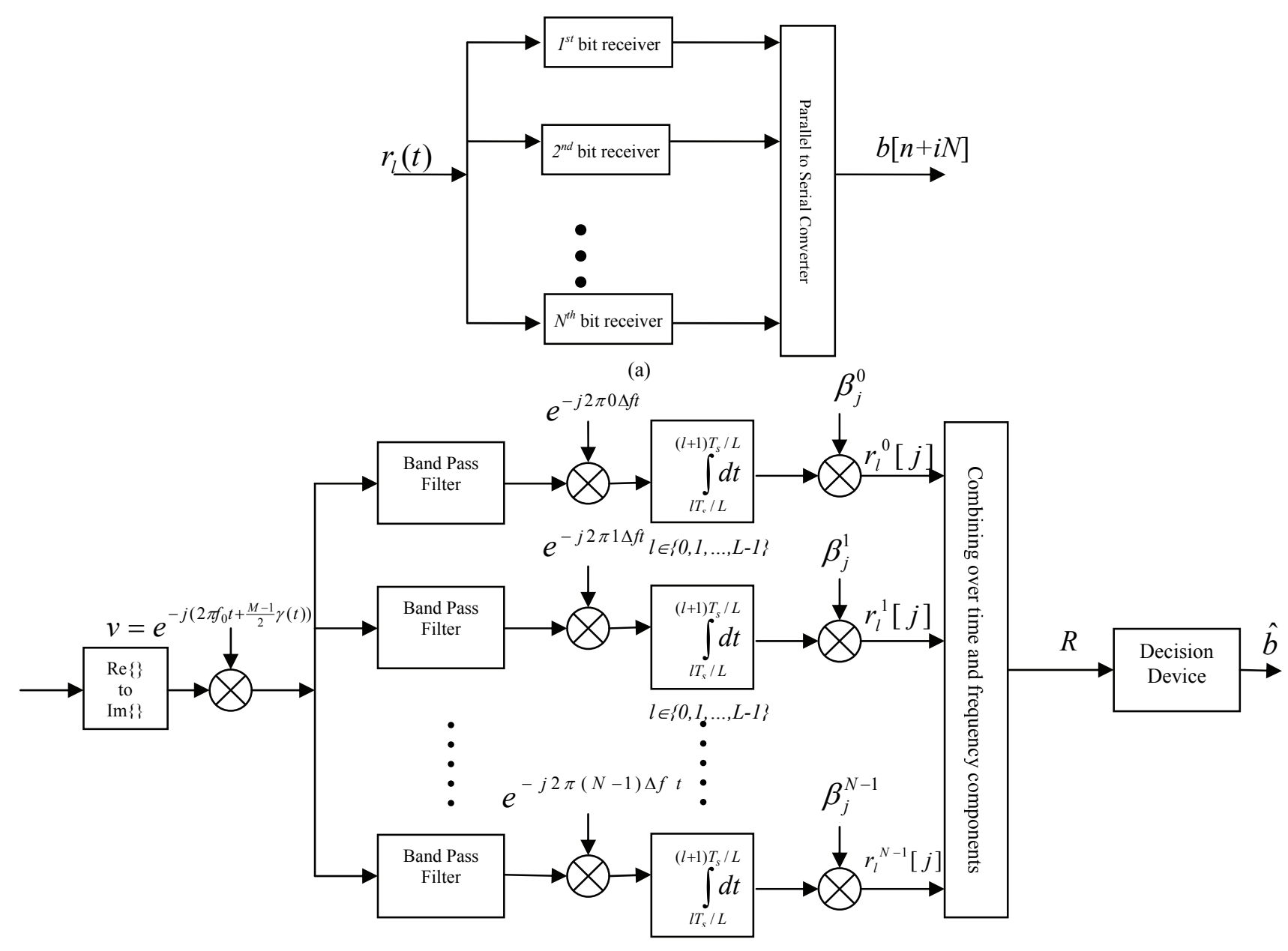

(b)

Figure 7. (a) BPS/MC-OFDM receiver structure (b) $j^{\text {th }}$ bit receiver.

(see [8]); and 3) Frequency-selective channel with fourfold frequency diversity over the entire bandwidth, i.e., in (14) $\Delta f /(\Delta f)_{c}=4 / N$.

Simulation results are presented in Figure 8. Simulations of BPS/OFDM with different combining techniques (Figure $8(\mathrm{a})$ ) in time domain show that MRC provides the best probability of error performance with the penalty of extra complexity. For single user case, MMSEC and MRC lead to similar performance results. MRC leads to a better performance as expected. Hence, MRC is chosen to exhibit the benefits of BPS merger with OFDM systems.

Simulations of MC-OFDM with different combining techniques (Figure $8(\mathrm{~b})$ ) reveal that MMSE combining provides the best performance. MRC leads to the worse performance, since in MC-OFDM systems each bit is transmitted over all sub-carriers utilizing orthogonality provided by Hadamard Walsh codes, and the orthogonality is destroyed when MRC is employed [24].

The top curve in Figure 8(c) represents the performance results for the benchmark system, i.e., traditional OFDM system with antenna array and without transmit diversity. The next curve shows MC-OFDM system performance with antenna array without BPS scheme. The BPS/MC-OFDM depicts a $15 \mathrm{~dB}$ and $5 \mathrm{~dB}$ improvement in performance at the probability-of-error of $10^{-3}$ compared to the traditional OFDM system and MC-OFDM system, respectively. This performance improvement is generated via time diversity created by beam pattern movement and is exploited using BPS/MC-OFDM receiver. It is also observed that BPS/OFDM performance is about $1 \mathrm{~dB}$ better than BPS/MC-OFDM system because MRC combining is the optimal combining when inter-bit-interference (IBI) is not available which lowersthe performance of BPS/MC-OFDM systems, comparatively. However, it has to be noted that BPS/MC-OFDM system mitigates the Peak-to-Average Power Ratio (PAPR) problem faced in OFDM systems.

Figure $8(d)$ shows the comparison of the coded MC-OFDM (MC-COFDM) with antenna array, with and without scanning. A $1 / 2$ - rate convolution code is considered and soft Viterbi algorithm is used for decoding. The simulation reveals that up to $6 \mathrm{~dB}$ and $5.5 \mathrm{~dB}$ improvement 


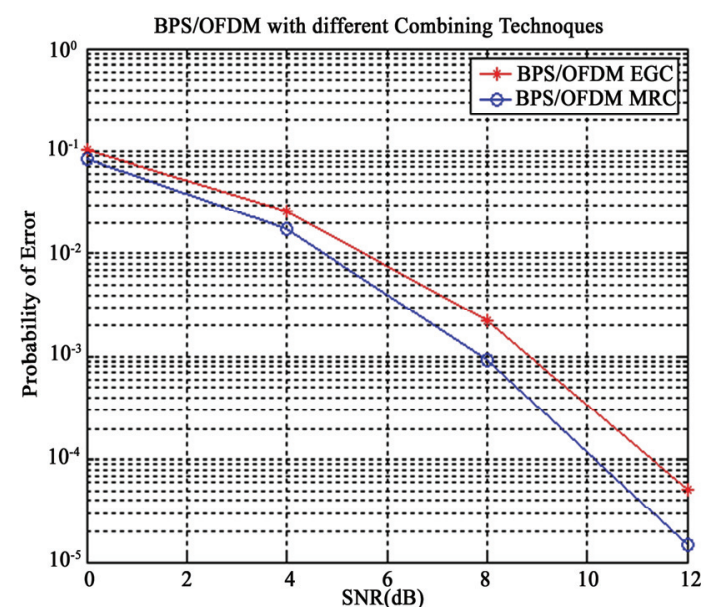

(a)

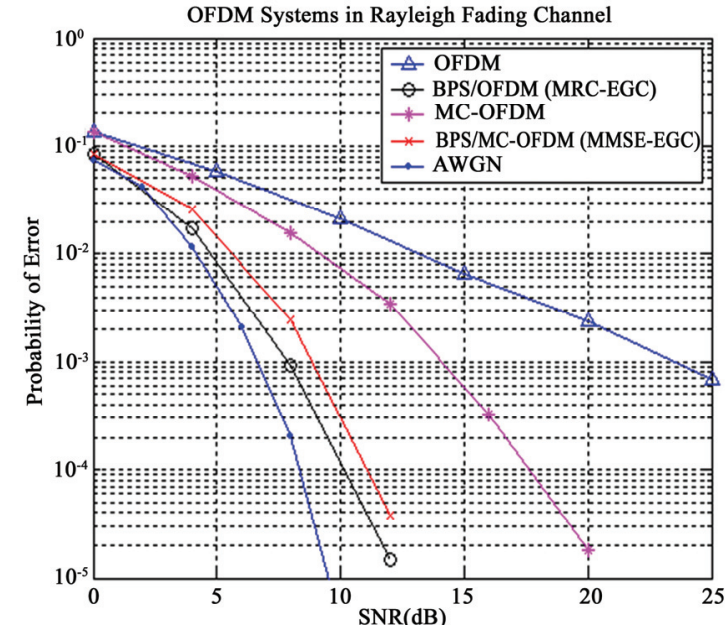

(c)

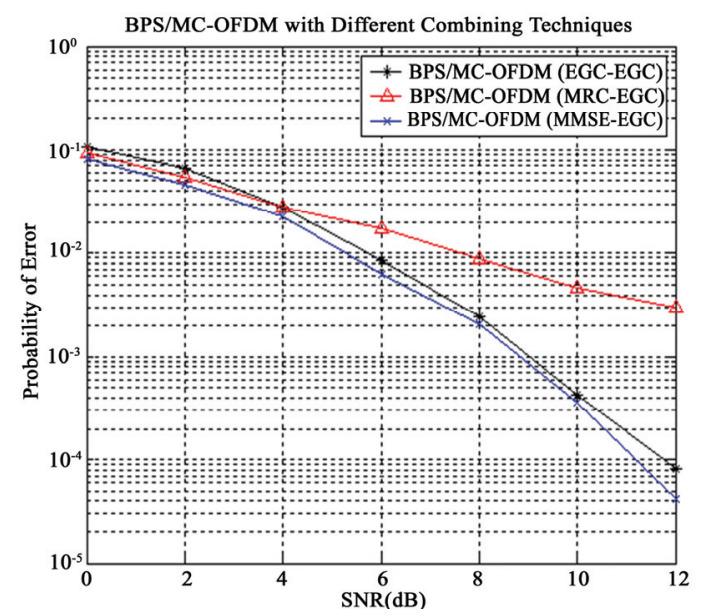

(b)

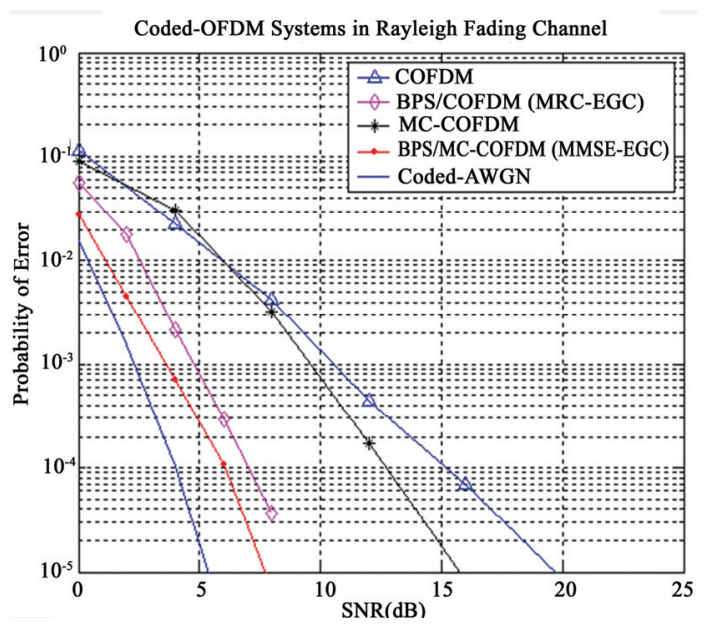

(d)

Figure 8. (a) Comparison of BPS/OFDM with different combining techniques; (b) Comparison of BPS/ MC-OFDM with different combining techniques; (c) Comparison of OFDM and MC-OFDM systems; (d) Comparison of coded OFDM and MC-OFDM systems.

in performance is achievable via BPS scheme at the probability-of-error of $10^{-3}$ comparing to COFDM and MCCOFDM systems. This clearly reinforce that BPS antenna arrays create time diversity that highly enhances the probability-of-error performance of MC-OFDM systems.

Despite the vast improvement in probability-of-error performance when applying forward error correction coding to MC-OFDM system, the decrease in the throughput makes it a less attractive solution. However, it is clear that BPS/MC-OFDM scheme (without coding) in Figure $8(\mathrm{c})$ offers a better performance at the probabilityof-error $10^{-3}$, compared to the traditional MC-COFDM in Figure 8(d), without sacrificing the throughput of the entire system.

Simulations of PAPR in Figure 9 shows that in general, MC-OFDM leads to a lower PAPR compared to OFDM systems. (e.g., $98 \%$ of the MC-OFDM transmissions demonstrate PAPR $<9$ while it is just $83 \%$ for traditional

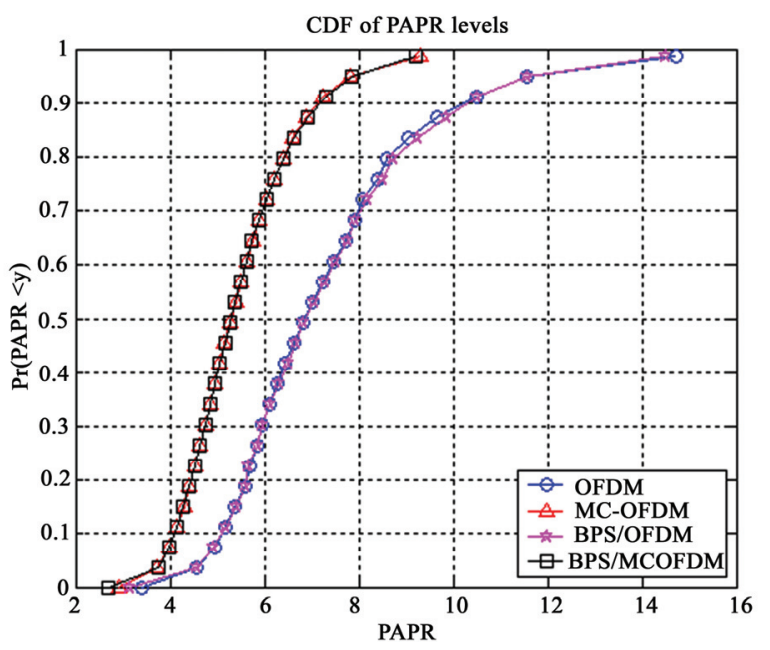

Figure 9. Comparison of PAPR of OFDM and BPS/MCOFDM. 
OFDM systems). The same results are generated for BPS/MC-OFDM systems. The simulations had proven that BPS and MC-OFDM merger is a superior technique capable of delivering high probability-of-error performance as well as reducing the PAPR problem in traditional OFDM systems. This introduces BPS merger with MCOFDM as a very attractive scheme for future generations of wireless communication systems.

\section{Conclusions}

The merger of BPS and MC-OFDM (BPS/MC-OFDM) was introduced and the PAPR together with the probability-of-error performance was studied. Time diversity is created using BPS scheme that highly enhances the probability-of-error performance of MC-OFDM systems. Moreover, the inherent unique transmission method of MC-OFDM lowers the PAPR problem compared to traditional OFDM system. This makes BPS/MC-OFDM merger a very competitive technique in wireless communications. The simulations performed also reveal that better performance is achievable via BPS/MC-OFDM (without coding) compared to MC-COFDM without reducing the throughput of the system inherent in MC-COFDM systems. The cost of deploying BPS/MCOFDM system is minimal due to the fact that the complexity of BPS system is mainly at the base station and the receiver complexity itself is minimal because the diversity components enter the receiver serially in time. Therefore, major improvement in performance at a minimal cost makes BPS/MC-OFDM a promising candidate for future wireless systems.

\section{References}

[1] R. V. Nee and R. Prasad, "OFDM for wireless multimedia communications," Artech House Publisher, Boston, MA. 2000.

[2] S. B. Weinstein and P. M. Ebert, "Data transmission by frequency-division multiplexing using the discrete fourier transform," IEEE Transactions on Communications, Vol. COM-19, No. 5, pp. 628-634, 1971.

[3] L. J. Cimini and Jr., "Analysis and simulation of a digital mobile channel using orthogonal frequency division multiplexing," IEEE Transactions on Communications, Vol. COM-33, No.7, pp. 665-675, 1985.

[4] R. W. Chang, "Orthogonal frequency division multiplexing," U.S. Patent 3, pp. 488-445, filed 1966, issued January $6,1970$.

[5] Z. Wang and G. B. Giannakis, "Wireless multicarrier communications: where fourier meets shannon," IEEE Signal Processing Magazine, Vol. 17, No. 3, pp. 29-48, May 2000.

[6] D. A. Wiegandt, Z. Wu, and C. R. Nassar, "Highthroughput, high-performance OFDM via pseudoorthogonal carrier interferometry spreading codes," IEEE Transactions on Communications, Vol. 51, No. 7, pp.
1123-1134, July 2003.

[7] B. Natarajan, C. R. Nassar, S. Shattil, Z. Wu, and M. Michelini, "High performance MC-CDMA via carrier interferometry codes," IEEE Transactions on Vehicular Technology, Vol. 50, No. 6, pp. 1344-1353, November 2001.

[8] P. K. Teh and S. A. Zekavat, "Merging multi-carrier OFDM and beam pattern scanning smart antennas: achieving low PAPR, high performance and high directionality," Proceedings 2003 Wireless Networking Symposium, The University of Texas at Austin, October 22-24, 2003.

[9] Y. G. Li, J. Chuang, and N. R. Sollenberger, "Transmit diversity for OFDM systems and its impact on high-rate data wireless networks," IEEE Journal of Selected Areas in Communications, Vol. 17, pp. 1233-1243, July 1999.

[10] D. Agarwal, V. Tarokh, A. Naguib, and N. Seshadri, "Space-time coded OFDM for high data rate wireless communication over wideband channels," in Proceedings 48th IEEE Vehicular Technology Conference, pp. 2232-2236, Ottawa, Canada, 18-21 May 1998.

[11] O. Norklit, P. C. F. Eggers, and J. B. Anderson, "Jitter diversity in multipath environments," IEEE 45th Vehicular Technology Conference, VTC'95, Vol. 2, pp. 853857, 25-28 July 1995.

[12] W. C. Wong, R. Steele, B. Glance, and D. Horn, "Time diversity with adaptive error detection to combat rayleigh fading in digital mobile radio," IEEE Transactions on Communications, Vol. COM-31, No. 3, pp. 378-387, March 1983.

[13] S. A. Zekavat and C. R. Nassar, "Antenna arrays with oscillating beam patterns: characterization of transmit diversity using semi-elliptic coverage geometric-based stochastic channel modeling," IEEE Transactions on Communications, Vol. 50, No. 10, pp. 1549-1556, October 2002.

[14] S. A. Zekavat and C. R. Nassar, "Achieving high capacity wireless by merging multi-carrier CDMA systems and oscillating-beam smart antenna arrays," IEEE Transactions on Vehicular Technology, Vol. 52, No. 4, pp. 772778, July 2003.

[15] S. A. Zekavat, C. R. Nassar, and S. Shattil, "Oscillating beam adaptive antennas and multi-carrier systems: Achieving transmit diversity, frequency diversity and directionality," IEEE Transactions on Vehicular Technology, Vol. 51, No. 5, pp. 1030-1039, September 2002.

[16] A. M. Sayeed and B. Azhang, "Joint multipath-doppler diversity in mobile wireless communications," IEEE Transactions on Communications, Vol. 47, No. 1, pp. 123-132, January 1999.

[17] J. C. Liberti, Jr., and T. S. Rappaport, "Smart antennas for wireless communications: Is-95 and third generation CDMA applications," Prentice Hall, PTR, Upper Saddle River, NJ, 1999.

[18] J. Fuhl, A. Kuchar, and E. Bonek, "Capacity increase in cellular PCS by smart antennas," IEEE 47th Vehicular Technology Conference, VTC'97, Vol. 3, No. 10, pp. 1962-1966, 1997. 
[19] P. K. Teh and S. A. Zekavat, "A merger of OFDM and antenna array Beam Pattern Scanning (BPS): achieving directionality and transmit diversity," Proceedings IEEE 37th Asilomar conference on Signals, Systems and Computers, Asilomar, CA, November 9-12, 2003.

[20] A. Kavak, "Adaptive antenna arrays for downlink capacity increase in third generation wireless CDMA," in IEEE Radio and Wireless Conference (RAWCON'01), pp. 77-80, Boston, MA, August 2001.

[21] A. F. Naguib, A. Paulraj, and T. Kailath, "Capacity improvement with base-station antenna arrays in cellular CDMA," IEEE Transactions on Vehicular Technology, Vol. 43, No. 3, pp. 691-698, August 1994.
[22] S. A. Zekavat, C. R. Nassar, and S. Shattil, "Smart antenna spatial sweeping for combined directionality and transmit diversity," Journal of Communications and Networks (JCN), Special Issue on Adaptive Antennas for Wireless Communications, Vol. 2, No. 4, pp. 325-330, December 2000.

[23] J. W. C. Jakes, "Microwave mobile communications," John Wiley, New York, NY, 1974.

[24] J. M. Auffray and J. F. Helard "Performance of multicarrier CDMA technique combined with space-time block coding over rayleigh channel," IEEE 7th International Symposium on Spread-Spectrum Technology, Vol. 2, pp. 348-352, 2-5 September 2002. 